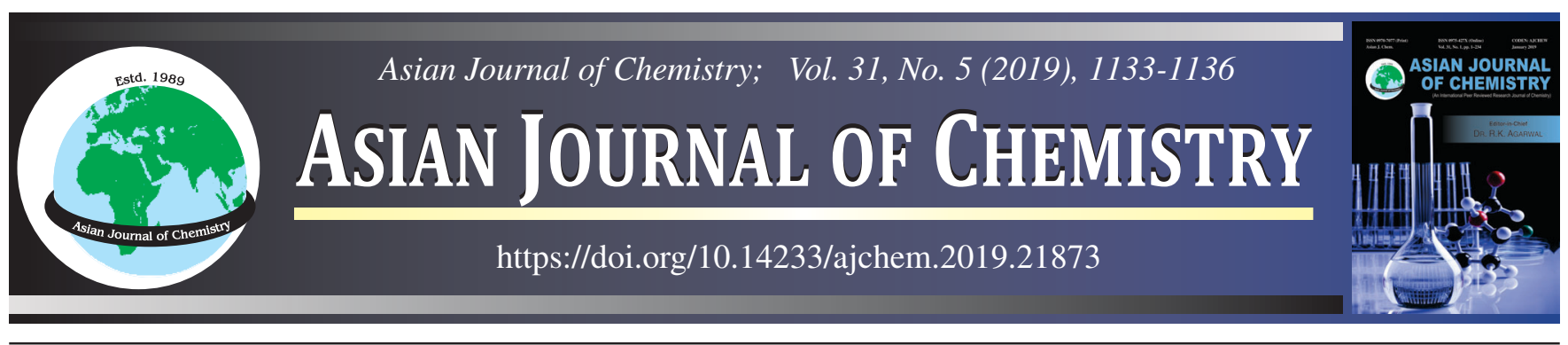

\title{
Microwave Assisted Efficient Synthesis of Flavone using ZnO Nanoparticles as Promoter under Solvent-Free Conditions
}

Pradip J. Unde, Nitin M. Thorat* and Limbraj R. Patil *

Post graduate Department of Chemistry, Maharaja Jivajirao Shinde Mahavidyalaya, Shrigonda-413701, India

*Corresponding authors: E-mail: nitin12.thorat@ gmail.com; limbrajp@gmail.com

Received: 12 December 2018; Accepted: 24 January 2019;

Published online: 28 March 2019;

AJC-19343

A simple and highly efficient protocol for synthesis of flavones from 1-(2-hydroxyphenyl)-3-aryl-1,3-propanediones in presence of ZnO nanoparticles as a promoter in thermal as well as microwave irradiation under solvent-free conditions have been demonstrated. The catalyst is inexpensive, stable, can be easily recycled/reused for several cycles with consistent activity and observed almost same yield confirming the stability of the catalyst. It is believed that the present approach will become an alternative route for the conventional reactions. Because in this protocol, yield is quite high, short reaction time, simple work up, catalyst can be recycled as well as it is free of any hazardous by-products formation during workup.

Keywords: Flavone, Nanocrystalline ZnO, Heterogeneous, Green Synthesis, Solvent-free synthesis.

ᄂ _ - _ - - - - _ - - - - - - - - - - - - - - - - - - - - - -

\section{INTRODUCTION}

Flavones are class of the flavonoids composing a 2-phenyl1-benzopyran-4-one backbone structure, which is present in fruits and vegetables ubiquitously. We consume flavanoids in our daily diet by inadvertently which have a beneficial impact on our health [1]. The flavones exhibited various biological activities based on the position and nature of the various substituent on the flavone structure. The flavones show a broad spectrum of biological activities due to their unique capacity to modulate different enzyme systems [2].

The flavones are active against infectious and metabolic diseases such as antiestrogenic, antimicrobial [3], anti-inflammatory, antioxidant [4], antiallergic, antitumor, vascular and cytotoxic [5] activities. In many natural products, flavone structure is frequently found such as luteolin and kaempferol. Due to their fascinating structural features, they exhibit array of biological activities, especially, anti-inflammatory, antimicrobial, antitumor and cytotoxic [6-11].

A verity of methods are used for synthesis of different flavones, which broadly can be classified into two groups viz. chalcones and 1,3-diketones as intermediates synthesized from $o$-hydroxyacetophenone. Most of the recently used methods for the synthesis of flavones depends on 1,3-diketones which is reported by Robinson and Venkataraman [12]. Several reported methods are available for synthesis of a flavone skeleton but one of the most commonly used methods are acid-catalyzed cyclization of various 1,3-diketone derived from 2-hydroxyacetophenones $[13,14]$. Various types of catalysts used for cyclization of 1-(2-hydroxyphenyl)-3-aryl-1,3-propanedione. But most of the reported ways of synthesis of flavones suffer from considerable restrictions such as use of harsh reaction conditions, prolonged reaction time, low yields, toxic and expensive catalysts/ reagents and solvents. In view of these restrictions, there is still a need for exploration of effortless, efficient, rapid, ecocompatible, and widely applicable routes for the synthesis of flavone owing to their immense synthetic and medicinal relevance. Also, due to the severe and burgeoning environmental regulations, organic chemists are call for the development of environmentally benevolent synthetic methodologies. Organic reactions under solvent-free conditions using microwave irradiation and conventional heating techniques has become a center of attraction for researchers over the years [15-17].

Recently, zinc oxide nanoparticles have received a significant attention because of its affordability, non-toxicity and has environmental advantages i.e. low corrosion, minimum completion time, easy transport, reuse of the catalyst, low waste and demolition of the catalyst [18]. Because of low coordi-

This is an open access journal, and articles are distributed under the terms of the Creative Commons Attribution-NonCommercial-ShareAlike 4.0 (CC BY-NC-SA 4.0) International License which allows readers to freely read, download, copy, distribute, print, search, or link to the full texts of its articles and to use them for any other lawful non-commercial purpose as long as the original source is duly acknowledged. 
nation sites and higher surface area, $\mathrm{ZnO}$ nanoparticles are considered to be more reactive. As the size decreases, the surface area of catalyst increases immensely which is useful for the greater catalytic activity. Some researchers have synthesized flavones from chalcones by using $\mathrm{ZnO}$ nanoparticles [19-21].

Due to huge number of advantages correlated with this eco-friendly nature, it has been used as a powerful catalyst for various organic transformations [22,23]. Hence, in present work in green synthesis of flavones by various methods, here we report an efficient and eco-friendly catalytic application of zinc oxide nanoparticles for synthesis of flavone from cyclodehydration of various 1,3-propanedione in microwave or conventional heating under solvent free conditions.

\section{EXPERIMENTAL}

All the chemical reactions were performed in corning glassware which is oven-dried. All chemicals and solvents were obtained from professional suppliers and used as such for further reactions. Thin-layer chromatography (TLC) was carried out on Merck silica gel plates, visualized with a UV lamp and stained in DNP solution and $\mathrm{KMnO}_{4}$. The standard methods are used for synthesis of 1-(2-hydroxyphenyl)-3-aryl-1,3-propanediones and their purities were established before utilization by melting point. Melting points were determined by open capillary tubes in paraffin oil bath. ${ }^{1} \mathrm{H}$ and ${ }^{13} \mathrm{C}$ NMR spectra were obtained as solutions in $\mathrm{CDCl}_{3}$ solvents. The standard ${ }^{1} \mathrm{H} \mathrm{NMR}(300 \mathrm{MHz})$ and ${ }^{13} \mathrm{C}$ NMR $(75 \mathrm{MHz})$ were recorded on a Varian Mercury spectrometer in $\mathrm{CDCl}_{3}$ solution. The tetramethylsilane was used as an internal standard. Perkin Elmer Model 1600 series FTIR instrument was used to recorded IR spectra. LC/MS (ES-API) instrument were used record Mass spectra. The QTOF Micromass Mass Spectrometer (electro spray ionization mode) was used for high-resolution mass spectra (HRMS). All the compounds synthesized in this article were previously reported. The spectroscopic and physical data is in matching with reported values.

\section{Synthesis of flavones using $\mathrm{ZnO}$ nanoparticles}

By conventional method: A mixture of substituted 1,3propanedione $(0.5 \mathrm{~g}, 2.08 \mathrm{mmol}, 1.0 \mathrm{eq}$.) and $\mathrm{ZnO}$ nanoparticles (1.0 eq.) was warmed in an oil bath at $100^{\circ} \mathrm{C}$ for $30 \mathrm{~min}$. After the finishing of reaction (TLC check), the product of reaction was allowed to cool at room temperature. To this reaction mixture $10 \mathrm{~mL}$ of water and $10 \mathrm{~mL}$ of ethyl acetate were added. Organic layer was isolated and the water layer was extracted in $(2 \times 10$ $\mathrm{mL}$ ) ethyl acetate. Mixed organic extract was dried over anhydrous sodium sulfate and concentrated in vacuum. Catalyst recycles by filtration of aqueous layer. The column chromatography is used for purification of crude product by using silica gel in hexane-ethylacetate solvent system to give the corresponding flavones (4a-l) in high yields.

Microwave method: A mixture of substituted 1,3-propanedione ( $0.5 \mathrm{~g}, 2.08 \mathrm{mmol}, 1.0$ eq.) and $\mathrm{ZnO}$ nanoparticles (1.0 eq.) was heated in a microwave oven for $15 \mathrm{~min}$. Same procedure used for purification of flavones as mentioned in the conventional heating method.

2-Phenyl-chromen-4-one (4a): m.p. $96-98^{\circ} \mathrm{C}$ [lit. 21]; IR $\left(\mathrm{KBr}, v_{\max }, \mathrm{cm}^{-1}\right): 756,1130,1568,1604,1645 ;{ }^{1} \mathrm{H}$ NMR
(300 MHz, $\left.\mathrm{CDCl}_{3}\right): \delta 6.81(\mathrm{~s}, 1 \mathrm{H}), 7.39(\mathrm{t}, J=7.8 \mathrm{~Hz}, 1 \mathrm{H})$, 7.46-7.56 (m, 4H), 7.65-7.70 (m, 1H), 7.88-7.91 (m, 2H), 8.21 $(\mathrm{d}, J=7.2 \mathrm{~Hz}, 1 \mathrm{H}) ;{ }^{13} \mathrm{C} \mathrm{NMR}\left(75 \mathrm{MHz}, \mathrm{CDCl}_{3}\right): \delta 107.3,117.9$, 123.7, 125.1, 125.5, 126.1, 128.9, 131.5, 131.6, 133.7, 156.1, 163.3, 178.3 ; LC-MS m/z: $223(\mathrm{M}+\mathrm{H})^{+}$.

2-(4-Methoxyphenyl)chromen-4-one (4b): m.p. 157$158^{\circ} \mathrm{C}$ [lit. 21]; IR (KBr, $\left.v_{\max }, \mathrm{cm}^{-1}\right): 767,1133,1465,1608$, $1649 ;{ }^{1} \mathrm{H} \mathrm{NMR}\left(300 \mathrm{MHz}, \mathrm{CDCl}_{3}\right): \delta 3.87(\mathrm{~s}, 3 \mathrm{H}), 6.80(\mathrm{~s}, 1 \mathrm{H})$, $7.01(\mathrm{~m}, 2 \mathrm{H}), 7.40(\mathrm{t}, J=7.2 \mathrm{~Hz}), 7.68(\mathrm{~m}, 1 \mathrm{H}), 7.53(\mathrm{~d}, J=8.1$ $\mathrm{Hz}), 7.88(\mathrm{~d}, 2 \mathrm{H}, J=7 \mathrm{~Hz}), 8.21(\mathrm{dd}, J=8.2 \& 2.1 \mathrm{~Hz}, 1 \mathrm{H})$; ${ }^{13} \mathrm{CNMR}\left(75 \mathrm{MHz}, \mathrm{CDCl}_{3}\right): \delta 55.4,105.9,114.4,117.9,123.7$, 123.9, 125.1, 125.6, 127.9, 133.6, 156.1, 162.4, 163.5, 178.5 ; LC-MS $m / z: 253(\mathrm{M}+\mathrm{H})^{+}$.

2-(4-Fluorophenyl)chromen-4-one (4c): m.p. 148-150 ${ }^{\circ} \mathrm{C}$ [lit. 21]; IR (KBr, $\left.v_{\max }, \mathrm{cm}^{-1}\right): 755,806,869,1134,1234$, $1467,1574,1608,1663 ;{ }^{1} \mathrm{H}$ NMR $\left(300 \mathrm{MHz}, \mathrm{CDCl}_{3}\right): \delta 6.79$ (s, $1 \mathrm{H}), 7.23(\mathrm{~m}, 2 \mathrm{H}), 7.47(\mathrm{t}, J=7.1 \mathrm{~Hz}, 1 \mathrm{H}), 7.58(\mathrm{~d}, J=7.1$ $\mathrm{Hz}, 1 \mathrm{H}), 7.72(\mathrm{~m}, 1 \mathrm{H}), 7.94(\mathrm{~m}, 2 \mathrm{H}), 8.24(\mathrm{~d}, J=7.2 \mathrm{~Hz}, 1 \mathrm{H})$; ${ }^{13} \mathrm{CNMR}\left(75 \mathrm{MHz}, \mathrm{CDCl}_{3}\right): \delta 107.1,116.6,117.0,123.9,125.5$, 126.0, 127.1, 128.1, 134.2, 156.0, 161.2, 162.6, 178.1; LCMS $m / z: 241(\mathrm{M}+\mathrm{H})^{+}$.

7-Methoxy-2-phenyl-chromen-4-one (4d): m.p. 109-110 ${ }^{\circ} \mathrm{C}$ [lit. 24]; IR (KBr, $\left.v_{\max }, \mathrm{cm}^{-1}\right): 767,908,1163,1450,1606$, 1626, 1647; ${ }^{1} \mathrm{H}$ NMR $\left(300 \mathrm{MHz}, \mathrm{CDCl}_{3}\right): \delta 3.91$ (s, 3H), 6.77 $(\mathrm{s}, 1 \mathrm{H}), 6.95(\mathrm{~m}, 2 \mathrm{H}), 7.51(\mathrm{~m}, 3 \mathrm{H}), 7.88(\mathrm{~m}, 2 \mathrm{H}), 8.11(\mathrm{~d}, J=8.7$ $\mathrm{Hz}, 1 \mathrm{H}) ;{ }^{13} \mathrm{CNMR}\left(75 \mathrm{MHz}, \mathrm{CDCl}_{3}\right): \delta 55.8,100.3,107.3,114.9$, 117.6, 126.0, 126.9, 128.9, 131.4, 131.6, 157.8, 162.9, 164.1, 177.7; LC-MS m/z: $253(\mathrm{M}+\mathrm{H})^{+}$.

7-Methoxy-2-(4-fluorophenyl)-chromen-4-one (4d): m.p. $172-173^{\circ} \mathrm{C}$ [lit. 21]; IR $\left(\mathrm{KBr}, v_{\max }, \mathrm{cm}^{-1}\right)$ : 781, 841, 1022, 1294, 1456, 1514, 1608, 1660; ${ }^{1} \mathrm{H}$ NMR (300 MHz, $\left.\mathrm{CDCl}_{3}\right)$ : $\delta 3.84(\mathrm{~s}, 3 \mathrm{H}), 6.41-6.48(\mathrm{~m}, 2 \mathrm{H}), 6.64(\mathrm{~s}, 1 \mathrm{H}), 7.15(\mathrm{t}, J=8.7$ $\mathrm{Hz}, 2 \mathrm{H}), 7.89(\mathrm{~m}, 1 \mathrm{H}), 7.90(\mathrm{~m}, 2 \mathrm{H}) ;{ }^{13} \mathrm{CNMR}\left(75 \mathrm{MHz}, \mathrm{CDCl}_{3}\right)$ : $\delta 55.6,101.3,108.1,110.1,115.7,116.1,128.9,128.9,130.1$, 163.4, 165.3, 165.9, 166.7, 174.8; LC-MS m/z: $271(\mathrm{M}+\mathrm{H})^{+}$.

7-Methoxy-2-(4-methoxyphenyl)chromen-4-one (4f): m.p. $194-195^{\circ} \mathrm{C}$ [lit. 24]; IR $\left(\mathrm{KBr}, v_{\max }, \mathrm{cm}^{-1}\right): 862,977,1186$, 1260, 1379, 1516, 1593, 1629; ${ }^{1} \mathrm{H}$ NMR (300 MHz, $\left.\mathrm{CDCl}_{3}\right)$ : $\delta 3.82(\mathrm{~s}, 3 \mathrm{H}), 3.86(\mathrm{~s}, 3 \mathrm{H}), 6.86(\mathrm{~m}, 2 \mathrm{H}), 6.60(\mathrm{~s}, 1 \mathrm{H}), 6.93(\mathrm{~d}$, $J=8.4 \mathrm{~Hz}, 2 \mathrm{H}), 7.77(\mathrm{~d}, J=8.4 \mathrm{~Hz}, 2 \mathrm{H}), 8.05(\mathrm{~d}, J=8.4 \mathrm{~Hz}$, $1 \mathrm{H}) ;{ }^{13} \mathrm{C}$ NMR $\left(75 \mathrm{MHz}, \mathrm{CDCl}_{3}\right): \delta 55.3,55.7,100.2,105.9$, 114.0, 114.2, 117.6, 126.7, 127.6, 157.7, 162.1, 162.8, 163.9, 177.6; LC-MS m/z: $283(\mathrm{M}+\mathrm{H})^{+}$.

6-Methoxy-2-phenyl-chromen-4-one (4g): m.p. 160-161 ${ }^{\circ} \mathrm{C}$ [lit. 24]; IR (KBr, $\left.v_{\max }, \mathrm{cm}^{-1}\right)$ : 658, 846, 1030, 1255, 1361, $1488,1618,1641 ;{ }^{1} \mathrm{H}$ NMR $\left(300 \mathrm{MHz}, \mathrm{CDCl}_{3}\right): \delta 3.89$ (s, 3H), $6.79(\mathrm{~s}, 1 \mathrm{H}), 7.28(\mathrm{dd}, J=6.6 \& J=3.5 \mathrm{~Hz}, 1 \mathrm{H}), 7.46-7.50(\mathrm{~m}$, $4 \mathrm{H}), 7.57(\mathrm{~d}, J=2.7 \mathrm{~Hz}, 1 \mathrm{H}), 7.88-7.91(\mathrm{~m}, 2 \mathrm{H}) ;{ }^{13} \mathrm{C} \mathrm{NMR}$ $\left(75 \mathrm{MHz}, \mathrm{CDCl}_{3}\right): \delta 55.8,104.7,106.7,119.4,123.6,124.4$, $126.1,128.8,131.4,131.7,150.8,156.9,163.0,178.2$; LCMS $m / z: 253(\mathrm{M}+\mathrm{H})^{+}$.

2-(4-Fluorophenyl)-6-methoxy-chromen-4-one (4h): m.p. $152-153^{\circ} \mathrm{C}$ [lit. 24]; IR $\left(\mathrm{KBr}, v_{\max }, \mathrm{cm}^{-1}\right)$ : 719, 910, 1024, 1168, 1488, 1620, 1661, 1727; ${ }^{1} \mathrm{H}$ NMR (300 MHz, $\left.\mathrm{CDCl}_{3}\right)$ : $\delta 3.90(\mathrm{~s}, 3 \mathrm{H}), 6.76(\mathrm{~s}, 1 \mathrm{H}), 7.18-7.30(\mathrm{~m}, 3 \mathrm{H}), 7.48(\mathrm{~d}, J=9.3$ $\mathrm{Hz}, 1 \mathrm{H}), 7.58(\mathrm{~d}, J=2.4 \mathrm{~Hz}, 1 \mathrm{H}), 7.92(\mathrm{~d}, J=9 \mathrm{~Hz}, 2 \mathrm{H}) ;{ }^{13} \mathrm{C}$ NMR (75 MHz, $\mathrm{CDCl}_{3}$ ): $\delta 55.9,104.8,116.1,116.4,119.4,123.8$, 124.4, 128.3, 128.4, 150.9, 157.0, 162.1, 166.3, 178.1; LC-MS 
$m / z: 271(\mathrm{M}+\mathrm{H})^{+} ;$HRMS $m / z[\mathrm{M}+\mathrm{H}]^{+}$: calcd for $\mathrm{C}_{16} \mathrm{H}_{11} \mathrm{O}_{3} \mathrm{~F}$ : 271.0692 .

6-Methoxy-2-(4-methoxyphenyl)chromen-4-one (4i): m.p. $195-196^{\circ} \mathrm{C}$ [lit. 24]; IR ( $\left.\mathrm{KBr}, v_{\max }, \mathrm{cm}^{-1}\right): 558,817,1014$, $1196,1268,1454,1584,1607,1647 ;{ }^{1} \mathrm{HNMR}\left(300 \mathrm{MHz}, \mathrm{CDCl}_{3}\right)$ : $\delta 3.88(\mathrm{~s}, 3 \mathrm{H}), 3.90(\mathrm{~s}, 3 \mathrm{H}), 6.73(\mathrm{~s}, 1 \mathrm{H}), 7.01(\mathrm{~d}, J=9.0 \mathrm{~Hz}$, 2H), $7.27(\mathrm{~m}, 1 \mathrm{H}), 7.58(\mathrm{~d}, J=3.0 \mathrm{~Hz}, 1 \mathrm{H}), 7.60(\mathrm{~d}, J=9.0$ $\mathrm{Hz}, 1 \mathrm{H}), 7.86(\mathrm{~d}, J=9.0 \mathrm{~Hz}, 2 \mathrm{H}) ;{ }^{13} \mathrm{C} \mathrm{NMR}\left(75 \mathrm{MHz}, \mathrm{CDCl}_{3}\right)$ : $\delta 55.4,55.9,104.8,105.4,114.4,119.3,123.5,124.1,124.4$, 127.8, 150.9, 156.8, 162.3, 163.1, 178.2; LC-MS m/z: 283 $(\mathrm{M}+\mathrm{H})^{+}$.

6-Fluoro-2-phenyl-chromen-4-one (4j): m.p. $128-129^{\circ} \mathrm{C}$ [lit. 21]; IR (KBr, $\left.v_{\max }, \mathrm{cm}^{-1}\right): 767,835,1176,1359,1570,1624$, $1660 ;{ }^{1} \mathrm{H}$ NMR $\left(300 \mathrm{MHz}, \mathrm{CDCl}_{3}\right): \delta 6.82(\mathrm{~s}, 1 \mathrm{H}), 7.39-7.46$ $(\mathrm{m}, 1 \mathrm{H}), 7.50-7.61(\mathrm{~m}, 4 \mathrm{H}), 7.85-7.93(\mathrm{~m}, 3 \mathrm{H}) ;{ }^{13} \mathrm{CNMR}(75 \mathrm{MHz}$, $\left.\mathrm{CDCl}_{3}\right): \delta 106.7,110.7,120.2,121.7,122.0,126.2,129.0,131.3$, 131.7, 152.3, 161.1, 163.1, 177.5 ; LC-MS m/z: $241(\mathrm{M}+\mathrm{H})^{+}$.

6-Bromo-2-phenyl-4H-chromen-4-one (4k): m.p. $176^{\circ} \mathrm{C}$; IR (KBr, $\left.v_{\max }, \mathrm{cm}^{-1}\right): 756,1130,1568,1604,1645 ;{ }^{1} \mathrm{H}$ NMR (300 MHz, $\left.\mathrm{CDCl}_{3}\right): \delta 7.12(\mathrm{~s}, 1 \mathrm{H}), 7.63(\mathrm{~m}, 3 \mathrm{H}), 7.83(\mathrm{~d}, J=3.2$ $\mathrm{Hz}, 1 \mathrm{H}), 8.11(\mathrm{dd}, J=10.8 \& 2.4 \mathrm{~Hz}, 1 \mathrm{H}), 8.13(\mathrm{~m}, 3 \mathrm{H}) ;{ }^{13} \mathrm{C}$ NMR $\left(75 \mathrm{MHz}, \mathrm{CDCl}_{3}\right): \delta 107.4,118.3,121.7,125.3,126.9$, 127.3, 129.6, 131.3, 132.5, 137.3, 155.1, 163.4, 176.3; HRMS $\mathrm{m} / z[\mathrm{M}+\mathrm{H}]^{+}$: calcd for $\mathrm{C}_{15} \mathrm{H}_{9} \mathrm{O}_{2} \mathrm{Br}: 300.9864$, found: 300.9867.

6-Chloro-2-phenyl-chromen-4-one (4l): m.p. 183-184 ${ }^{\circ} \mathrm{C}$ [lit. 24]; IR $\left(\mathrm{KBr}, v_{\max }, \mathrm{cm}^{-1}\right)$ : 682, 908, 1132, 1307, 1438, 1457, 1567, 1601, 1651; ${ }^{1} \mathrm{H}$ NMR (300 MHz, $\left.\mathrm{CDCl}_{3}\right): \delta 6.82$ $(\mathrm{s}, 1 \mathrm{H}), 7.50-7.56(\mathrm{~m}, 4 \mathrm{H}), 7.60-7.65(\mathrm{~m}, 1 \mathrm{H}), 7.88-7.90(\mathrm{~m}, 2 \mathrm{H})$, $8.17(\mathrm{~d}, J=2.3 \mathrm{~Hz}, 1 \mathrm{H}) ;{ }^{13} \mathrm{C} \mathrm{NMR}\left(75 \mathrm{MHz}, \mathrm{CDCl}_{3}\right): \delta 107.3$, 119.7, 124.7, 125.1, 126.3, 129.1, 131.1, 131.2, 131.8, 133.0, 154.4, 163.7, 177.1; LC-MS m/z: $257(\mathrm{M}+\mathrm{H})^{+}$.

\section{RESULTS AND DISCUSSION}

Optimization of reaction conditions: To study the catalytic activity in synthesis of flavones from 1-(2-hydroxyphenyl)-3aryl-1,3-propanedione (3a) was chosen as a representation reaction. For this, we first examined the synthesis of 2-phenyl$4 H$-chromen-4-one (4a) in the presence of 0.1 equivalent of $\mathrm{ZnO}$ nanoparticles $(0.208 \mathrm{mmol}, 0.012 \mathrm{~g})$. While performing conventional heating even after $60 \mathrm{~min}$, the desired product 4a was produced in a low yield (entry 1 , Table-1). Using 0.2 $(0.442 \mathrm{mmol}, 0.034 \mathrm{~g})$ and $0.4(0.832 \mathrm{mmol}, 0.068 \mathrm{~g})$ equivalents of $\mathrm{ZnO}$ nanoparticles gives the desired amount of product in moderate yields (entries 2 and 3, Table-1,), while using $0.6(0.10 \mathrm{~g}, 1.25 \mathrm{mmol})$ and $0.8(0.166 \mathrm{mmol}, 0.14 \mathrm{~g})$ equivalents of $\mathrm{ZnO}$ nanoparticles, there was insignificant raise in the yield of the desired product (entry 4 and 5, Table-1).

Finally, it was observed that $1.0(0.16 \mathrm{~g}, 2.08 \mathrm{mmol})$ equivalent of $\mathrm{ZnO}$ nanoparticles was the most suitable amount of catalyst for achieving the desired product (Table-1, entry 6). By applying the same approach under microwave heating, it was also observed that the time taken for completion of this reaction was now reduced to half of the time taken by convention heating (entry 1- 6, Table-1).

Regeneration of $\mathrm{ZnO}$ nanoparticles: The $\mathrm{ZnO}$ nanoparticles catalyst reused and recovered by filtration of reaction mixture. It was used for subsequent experiments (up to five times) under the same reaction conditions. It was also observed that
TABLE-1

EFFECT USE OF DIFFERENT AMOUNT OF CATALYST ON THE SYNTHESIS OF FLAVONES ${ }^{a}$
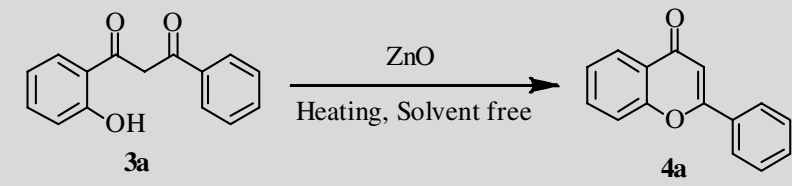

\begin{tabular}{cccc|cc}
\hline & & \multicolumn{2}{c|}{ Heating in oil bath } & \multicolumn{2}{c}{ Heating in microwave } \\
\cline { 3 - 6 } Entry & Catalyst & $\begin{array}{c}\text { Time } \\
(\mathrm{min})\end{array}$ & $\begin{array}{c}\text { Yield } \\
(\%)\end{array}$ & $\begin{array}{c}\text { Time } \\
(\mathrm{min})\end{array}$ & $\begin{array}{c}\text { Yield } \\
(\%)\end{array}$ \\
\hline 1 & 0.1 eq. & 60 & 20 & 60 & 22 \\
2 & 0.2 eq. & 30 & 32 & 15 & 38 \\
3 & 0.4 eq. & 30 & 44 & 15 & 48 \\
4 & 0.6 eq. & 30 & 66 & 15 & 70 \\
5 & 0.8 eq. & 30 & 74 & 15 & 78 \\
6 & 1.0 eq. & 30 & 80 & 15 & 88 \\
\hline
\end{tabular}

${ }^{a}$ Reagent: 1-(2-hydroxyphenyl)-3-phenylpropane-1, 3-dione (0.5 g)

yields of the product remained comparable to subsequent experiments (Fig. 1). Thus, it is finally inferred that the reusability and recyclability of the catalyst remain constant without significant loss of activity.

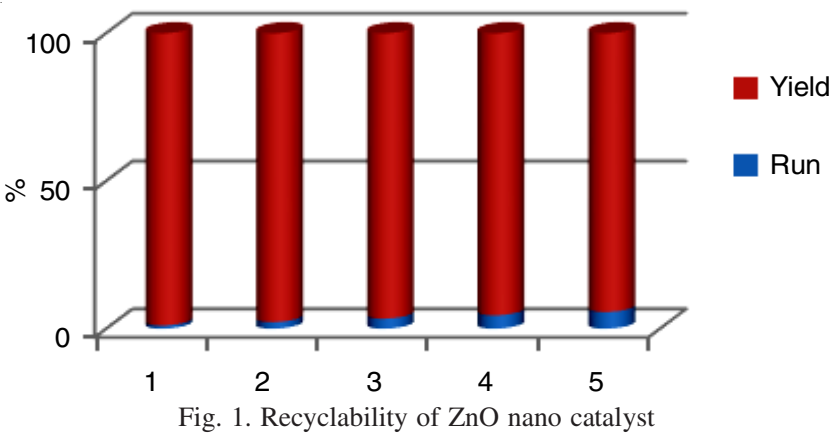

Next, to explore the diversity of this methodology, various 1-(2-hydroxyphenyl)-3-aryl-1,3-propanediones were tested under the above reaction condition. Results showed that cyclization of various 1-(2-hydroxyphenyl)-3-aryl-1,3-propanediones proceeded smoothly to completion within a short reaction time to give the corresponding flavones in good to excellent yields under both heating conditions (entry 1-10, Table-2).

\section{Conclusion}

In summary, a simple, rapid and environmentally benign protocol is developed in which $\mathrm{ZnO}$ nanoparticles as an effective promoter for synthesis of various flavones under solvent freeconditions. Microwave assisted reactions are appeared to be slightly more energy efficient as it requires less time for completion as compared to the traditionally heated reaction. This protocol is more straight-forward because of mild reaction conditions, economically viable and eco-friendly, thus providing a very efficient alternative to traditional processes.

\section{ACKNOWLEDGEMENTS}

The authors are thankful to the Council of BCUD, Savitribai Phule Pune University, Pune, India for financial assistance and The Principal, Dr. D.K. Mhaske, Maharaja Jivajirao Shinde Mahavidyalaya, Shrigonda, India for additional support for this research project. 
TABLE-2

ZnO NANOPARTICLE PROMOTED SYNTHESIS OF FLAVONES FROM 1-(2-HYDROXYPHENYL)-3-ARYL-1,3-PROPANEDIONE<smiles>[R]C(=O)CC(=O)c1cc([R])c([R])cc1O</smiles>

3

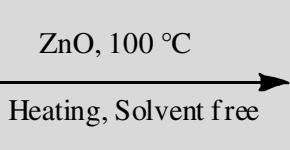

Heating, Solvent free<smiles>[R5]c1cc(=O)c2ccccc2o1</smiles>

4

\begin{tabular}{|c|c|c|c|c|c|c|}
\hline \multirow{2}{*}{ Entry } & \multirow{2}{*}{ Compd. } & \multirow{2}{*}{$\mathrm{R}_{1}$} & \multirow{2}{*}{$\mathrm{R}_{2}$} & \multirow{2}{*}{$\mathrm{R}_{3}$} & Conventional heating $^{\mathrm{b}}$ & Microwave heating $^{\mathrm{c}}$ \\
\hline & & & & & Yield $^{\mathrm{d}}(\%)$ & Yield $^{\mathrm{d}}(\%)$ \\
\hline 1 & $4 a$ & $\mathrm{H}$ & $\mathrm{H}$ & $\mathrm{Ph}$ & 80 & 88 \\
\hline 2 & $4 b$ & $\mathrm{H}$ & $\mathrm{H}$ & $4-\mathrm{MeOC}_{6} \mathrm{H}_{4}$ & 81 & 87 \\
\hline 3 & $4 c$ & $\mathrm{H}$ & $\mathrm{H}$ & 4- $\mathrm{FC}_{6} \mathrm{H}_{4}$ & 78 & 88 \\
\hline 4 & 4d & $\mathrm{OMe}$ & $\mathrm{H}$ & $\mathrm{Ph}$ & 79 & 86 \\
\hline 5 & $4 e$ & $\mathrm{OMe}$ & $\mathrm{H}$ & $4-\mathrm{FC}_{6} \mathrm{H}_{4}$ & 80 & 88 \\
\hline 6 & $4 f$ & $\mathrm{OMe}$ & $\mathrm{H}$ & 4- $\mathrm{MeOC}_{6} \mathrm{H}_{4}$ & 80 & 87 \\
\hline 7 & $4 g$ & $\mathrm{H}$ & $\mathrm{OMe}$ & $\mathrm{Ph}$ & 81 & 88 \\
\hline 8 & $4 h$ & $\mathrm{H}$ & $\mathrm{OMe}$ & 4- $\mathrm{FC}_{6} \mathrm{H}_{4}$ & 80 & 86 \\
\hline 9 & $4 \mathbf{i}$ & $\mathrm{H}$ & $\mathrm{OMe}$ & 4- $\mathrm{MeOC}_{6} \mathrm{H}_{4}$ & 81 & 89 \\
\hline 10 & $\mathbf{4 j}$ & $\mathrm{H}$ & $\mathrm{F}$ & $\mathrm{Ph}$ & 78 & 86 \\
\hline 11 & $4 k$ & $\mathrm{H}$ & $\mathrm{Br}$ & $\mathrm{Ph}$ & 79 & 85 \\
\hline 12 & 41 & $\mathrm{H}$ & $\mathrm{Cl}$ & $\mathrm{Ph}$ & 80 & 87 \\
\hline
\end{tabular}

${ }^{\mathrm{a}}$ Reagents: (1) (0.5 g.) various 1, 3-propanediones (3a-3m, 1.0 eq.), (2) $\mathrm{ZnO}$ nanoparticle (1.0 eq.). ${ }^{\mathrm{b}} 30 \mathrm{~min}$ in conventional heating. ${ }^{\mathrm{c}} 15 \mathrm{~min}$ in microwave. ${ }^{\mathrm{d}}$ Isolated yields.

\section{CONFLICT OF INTEREST}

The authors declare that there is no conflict of interests regarding the publication of this article.

\section{REFERENCES}

1. V.C. Blank, C. Poli, M. Marder and L.P. Roguin, Bioorg. Med. Chem. Lett., 14, 133 (2004);

https://doi.org/10.1016/j.bmcl.2003.10.029.

2. A.K. Verma and R. Pratap, Nat. Prod. Rep., 27, 1571 (2010); https://doi.org/10.1039/c004698c.

3. T.P.T. Cushnie and A. Lamb, Int. J. Antimicrob. Agents, 26, 343 (2005); https://doi.org/10.1016/j.ijantimicag.2005.09.002.

4. B. Havsteen, Biochem. Pharmacol., 32, 1141 (1983); https://doi.org/10.1016/0006-2952(83)90262-9.

5. E. Middleton Jr. and K. Chithan, ed.: J.B. Harborne, The Flavonoids: Advances in Research Since 1986; Chapman \& Hall: London, pp. 619652 (1993).

6. S. Martens and A. Mithofer, Phytochemistry, 66, 2399 (2005); https://doi.org/10.1016/j.phytochem.2005.07.013.

7. $\quad$ N.C. Veitch and R.J. Grayer, Nat. Prod. Rep., 25, 555 (2008); https://doi.org/10.1039/b718040n.

8. A. Crozier, I.B. Jaganath and M.N. Clifford, Nat. Prod. Rep., 26, 1001 (2009);

https://doi.org/10.1039/b802662a.

9. U. Sequin, The Antibiotics of the Pluramycin Group (4H-Anthra [1,2-b]pyran Antibiotics), In: Progress in the Chemistry of Organic Natural Products, Springer: Vienna, vol. 50, pp. 57-122 (1986).

10. M.R. Hansen and L.H. Hurley, Acc. Chem. Res., 29, 249 (1996); https://doi.org/10.1021/ar950167a.

11. J. Gabrielska, M. Soczyñska-Kordala and S. Przestalski, J. Agric. Food Chem., 53, 76 (2005);

https://doi.org/10.1021/jf0401120.
12. J. Allan and R. Robinson, J. Chem. Soc., 125, 2192 (1924); https://doi.org/10.1039/CT9242502192.

13. W. Baker, J. Chem. Soc., 1381 (1933); https://doi.org/10.1039/jr9330001381.

14. H.S. Mahal and K. Venkataraman, J. Chem. Soc., 1767 (1934); https://doi.org/10.1039/jr9340001767.

15. N.M. Thorat, S.R. Kote and S.R. Thopate, Lett. Org. Chem., 11, 601 (2014); https://doi.org/10.2174/157017861108140613163214.

16. L. Rout, T.K. Sen and T. Punniyamurthy, Angew. Chem. Int. Ed., 46, 5583 (2007); https://doi.org/10.1002/anie.200701282.

17. B.M. Choudary, M.L. Kantam, K.V.S. Ranganath, K. Mahendar and B. Sreedhar, J. Am. Chem. Soc., 126, 3396 (2004); https://doi.org/10.1021/ja038954n.

18. F.M. Moghaddam, H. Saeidian, Z. Mirjafary and A. Sadeghi, J. Iran Chem. Soc., 6, 317 (2009); https://doi.org/10.1007/BF03245840.

19. S. Chand and J.S. Sandhu, Indian J. Chem., 54B, 1350 (2015).

20. D. Mulugeta, B. Abdisa, A. Belay and M. Endale, Chem. Mater. Res., 10, 1 (2018)

21. C. Tamuly, I. Saikia, M. Hazarika, M. Bordoloi, N. Hussain, M.R. Das and K. Deka, RSC Adv., 5, 8604 (2015); https://doi.org/10.1039/C4RA14225J.

22. N. Shantikumar, A. Sasidharan, V.V. Divya Rani, D. Menon, S. Nair, K. Manzoor and S. Raina, Mater. Sci. Mater. Med., 20, 235 (2009); https://doi.org/10.1007/s10856-008-3548-5.

23. M. Premanathan, K. Karthikeyan, K. Jeyasubramanian and M. Govindasamy, Nanomedicine, 7, 184 (2011); https://doi.org/10.1016/j.nano.2010.10.001.

24. N.M. Thorat, R.A. Dengale, S.R. Thopate and S.V. Rohokale, Lett. Org. Chem., 12, 574 (2015); https://doi.org/10.2174/1570178612666150624172950. 